\title{
ZIF-8 membrane: the synthesis technique and nanofiltration application
}

\author{
Dayang Norafizan Awang Chee ${ }^{1,2} \cdot$ Farhana Aziz $^{1}$ Mohamed Afizal Mohamed Amin ${ }^{1,3} \cdot$ Ahmad Fauzi Ismail $^{1}[$
}

Received: 1 July 2021 / Accepted: 9 December 2021

(c) Qatar University and Springer Nature Switzerland AG 2021

\begin{abstract}
Membrane technology for water treatment is growing due to the increased demand for clean water and the stringent standard for water quality. Nanofiltration (NF) is widely used in membranes for water treatment due to lower energy consumption and higher flux rates. The application was not limited to only water treatment, yet NF showed excellent potential for solvent nanofiltration application. The utilization of MOF materials for the preparation of NF membranes to produce composite membranes has attracted more attention among researchers due to the advantages offered by the material. Zeolitic imidazolate framework-8 (ZIF-8) membrane has been considered a promising MOF membrane with its capabilities and promising performance due to the small aperture size of the compounds and the stability towards harsh chemical condition. The purpose of this review is to present the synthesis approaches on the preparation of ZIF-8 membranes and the application of ZIF-8 membrane for NF application. The challenges and prospects of ZIF-8 membranes are also discussed to further stimulate the development and the application of ZIF-8 membrane for NF.
\end{abstract}

Keywords MOF · ZIF- $8 \cdot$ Membrane $\cdot$ Nanofiltration $\cdot$ Organic solvent nanofiltration $\cdot$ Water treatment

\section{Introduction}

Nanofiltration has been recognized in the late 1980s with the properties that resemble ultrafiltration (UF) and reverse osmosis (RO). The molecular weight cut-off of the nanofiltration membrane is $300-500 \mathrm{Da}$, with a pore size typically of $1 \mathrm{~nm}$. Nanofiltration membrane has a great potential in the separation of inorganic salts and small organic dye molecules. The difference between NF and RO is that the NF membranes have low rejection on the monovalent ions, yet

Ahmad Fauzi Ismail

afauzi@utm.my

1 Advanced Membrane Technology Research Centre (AMTEC), School of Chemical and Energy Engineering, Faculty of Engineering, Universiti Teknologi Malaysia, Johor Bahru, 81310 Johor Darul Takzim, Malaysia

2 Faculty of Resource Science and Technology, Universiti Malaysia Sarawak, 94300 Kota Samarahan, Sarawak, Malaysia

3 Department of Chemical Engineering and Energy Sustainability, Faculty of Engineering, Universiti Malaysia Sarawak, 94300 Kota Samarahan, Sarawak, Malaysia having high rejection in divalent ions with higher flux in comparison with the RO membranes. The NF system is also able to operate at modest pressure. These attributes have made the NF competitive enough to compare with the traditional approach for water and wastewater treatment application, pharmaceutical and biological processes. Recently, the emerging of the metal-organic framework (MOF) has contributed much to the development of NF membranes.

MOFs are a type of porous, zeolite-like material that combines the qualities of metal and organic compound properties. From the beginning of the twenty-first century, MOF has been studied for their preparation methods and application for the various fields including separation. The MOFs have been introduced as nanofillers in membranes [1], pure MOF membranes [2] and additives in thin-film membranes [3]. Based on their promising potential, in the past 10 years, various outstanding MOF membranes have been successfully fabricated and used including nanofiltration application.

The nomenclature of MOF material is quite random. For example, the zeolitic imidazolate frameworks (ZIFs), a subclass of MOF has almost the same properties with zeolites, in term of its crystallinity, microporosity, large surface area and 